\title{
Short communication: Aquaporin-7 mRNA in adipose depots of primiparous and pluriparous dairy cows: Long-term physiological and conjugated linoleic acid-induced changes
}

\author{
H. Sauerwein, ${ }^{* 1}$ B. Saremi, ${ }^{*}$ J. Pappritz, $†$ D. von Soosten, $\dagger$ U. Meyer, $†$ S. Dänicke, $†$ and M. Mielenz ${ }^{* 2}$ \\ *Institute of Animal Science, Physiology \& Hygiene Unit, University of Bonn, Katzenburgweg 7 - 9, 53115 Bonn, Germany \\ †Institute of Animal Nutrition, Friedrich-Loeffler-Institute (FLI), Federal Research Institute for Animal Health, 38116 Braunschweig, Germany
}

\begin{abstract}
Aquaglyceroporins act as channel proteins and regulate water and glycerol exchange through cell membranes. The aquaglyceroporin aquaporin-7 (AQP7) is abundantly expressed in adipose tissue (AT) and regulates the release of glycerol produced by lipolysis. We aimed to investigate the expression of AQP7 mRNA during lactation in subcutaneous (s.c.) and visceral (v.c.) adipose depots of primiparous and pluriparous dairy cows. In 2 independent experiments, Holstein cows were supplemented with conjugated linoleic acids (CLA) or a control (CON) fat supplement at $100 \mathrm{~g} / \mathrm{d}$. Pluriparous cows were supplemented starting with the first day in milk (DIM) up to 182 DIM and biopsies from s.c. AT were collected at d -21, 1, 21, 70, 105182 196,224 , and 252 relative to calving (CLA $=11$; CON $=10)$. Samples from 3 s.c. and v.c. adipose depots were investigated in primiparous cows $(\mathrm{n}=25)$ receiving the supplements from 1 DIM until slaughter at 1, 42, or 105 DIM. The AQP7 mRNA abundance decreased from d -21 to 1 in s.c. AT of pluriparous cows without further increase to d 252 of lactation. In primiparous cows of the CON group, the AQP7 mRNA abundance increased from 1 to 105 DIM in s.c. AT from the tail head and in mesenteric AT. In retroperitoneal AT, the only depot for which a significant decrease in mass was observed with DIM, AQP7 mRNA abundance was greater at 42 and 105 than 1 DIM. Comparing the different fat depots, retroperitoneal AT had the highest and mesenterial AT had the lowest AQP7 mRNA abundance, but no general difference was observed between v.c. and s.c. fat depots. The values were not affected by CLA treatment with the exception of mesenteric AT, for which lower AQP7 mRNA abundance values were recorded in the CLA than in the CON group.
\end{abstract}

Received November 8, 2012.

Accepted April 1, 2013.

${ }^{1}$ Corresponding author: sauerwein@uni-bonn.de

${ }^{2}$ Present address: Institute of Nutritional Physiology "Oskar Kellner," Leibniz Institute for Farm Animal Biology (FBN), WilhelmStahl-Allee 2, 18196 Dummerstorf, Germany.
The longitudinal characterization of the AQP7 mRNA expression profile throughout lactation revealed differences between primiparous and pluriparous cows, with an increase of AQP7 mRNA abundance up to 105 DIM only in the primiparous cows. Due to a lack of CLA effects in pluriparous cows and the limitation to just one fat depot in primiparous cows, a modulatory effect of CLA on AQP7 mRNA abundance in dairy cows is not supported by our study.

Key words: aquaporin-7, conjugated linoleic acid, adipose tissue, dairy cow

\section{Short Communication}

Aquaglyceroporins (AQP) comprise a subfamily of aquaporins that allow the movement of water and glycerol through cell membranes. In adipose tissue (AT), AQP3, AQP7, and AQP9 are expressed, but only AQP7 was recently reported to be detectable at the protein level in human AT (Lebeck et al., 2012). Aquaporin-7 serves as a glycerol channel (Kishida et al., 2000), is predominantly found in the cytoplasm upon lipid droplets (Rodríguez et al., 2011b), and is considered as the main gateway for the delivery of fat-derived glycerol (Rodríguez et al., 2011a).

Glycerol is an important metabolite for the control of fat accumulation as the carbon backbone of triacylglycerides (TAG) and also for glucose homeostasis because glycerol forms a substrate for gluconeogenesis mainly during fasting. Under lipogenic conditions, adipocytes synthesize TAG by esterification of FFA and glycerol3-phosphate (G3P); glycerol from lipolysis cannot be directly phosphorylated to G3P, as AT has only negligible levels of glycerol kinase (Chilliard, 1993). The generation of G3P may occur from glucose, but de novo synthesis from sources such as lactate and pyruvate, in a process termed glyceroneogenesis, has also been described at least in rodent AT, where phosphoenolpyruvate carboxykinase and pyruvate dehydrogenase kinase 4 have been identified as essential components of the glyceroneogenic enzymatic machinery (Reshef et al., 2003). For lipolysis-induced glycerol release from adipo- 
cytes, AQP7 appears to be the main channel (Kishida et al., 2000) and therefore prevents an acute rise in intracellular osmotic pressure when glycerol is rapidly produced during lipolysis (Maeda, 2012). Fasting has been demonstrated to increase AQP7 mRNA (Kishida et al., 2001a); however, classic lipolytic stimuli (e.g., catecholamines) induce a translocation of AQP7 to the plasma membrane and, under long-term stimulation, may also reduce AQP7 expression (Fasshauer et al., 2003; Rodríguez et al., 2011b). For insulin as a further regulator of AQP7 expression, conflicting results were obtained in different species. Insulin repressed AQP7 mRNA abundance in murine 3T3-L1 adipocytes (Kishida et al., 2001b), but increased the protein expression in differentiated omental adipocytes from obese normoglycemic humans (Rodríguez et al., 2011b). Studies in obese humans suggest that the regulation of aquaglyceroporins in AT is more closely related to decreased insulin sensitivity (IS) than to obesity (Rodríguez et al., 2011b). In dairy cows, the energy requirements for milk yield and maintenance exceed energy intake by voluntary feed intake during the first weeks of lactation. The prioritization of milk secretion at the cost of body reserves is achieved by reduced IS in peripheral organs, except the mammary gland (Bell and Bauman, 1997), and by an increase in gluconeogenesis along with a decrease in glucose oxidation (Bennink et al., 1972; Bell, 1995) to support the glucose-derived lactose output with milk. Together with lipolytic stimuli, this results in the breakdown of TAG in AT and in consequence to the release of FFA and glycerol into the bloodstream. As recently reviewed (Aschenbach et al., 2010), the portion of glycerol used for gluconeogenesis is generally low $(0.5-3 \%)$, but increases during energy deficiency and lipid mobilization. Depending on the extent and duration of lipolysis, and also the coping capability of the individual animal, metabolic disturbances, such as ketosis and fatty liver, may occur in early lactation. Reducing the energy output via milk by decreasing the milk fat content through feed supplements containing conjugated linoleic acids (CLA) may attenuate the negative energy balance during this period. In monogastric species, dietary CLA supplements have been demonstrated to reduce body fat, but the mechanisms of action are not fully clarified. Peroxisome proliferatoractivated receptor- $\gamma$ (PPAR $\gamma$ ) functioning as a receptor for CLA has been identified as a key component for the cellular actions of CLA (Bensinger and Tontonoz, 2008) and a PPAR $\gamma$ response element in the AQP7 promoter region has been described (Kishida et al., 2001b). In dairy cows receiving CLA supplements, body fat seems largely unaffected, as evident from various indicators for body fat content and lipolysis (e.g., Baumgard et al., 2002; Kay et al., 2006). For adipocyte size, we recently observed a decrease with CLA treatment in 5 of 6 different visceral (v.c.) and subcutaneous (s.c.) fat depots (Akter et al., 2011). With this background we aimed to test the hypothesis that AQP7 expression will be changed by lactation-induced lipolysis in a fat depot-specific manner and that CLA will interfere in this.

We used tissue samples from 2 animal experiments in which the animals were fed either $100 \mathrm{~g} / \mathrm{d}$ of CLA (Lutrell Pure, BASF, Ludwigshafen, Germany) containing equal portions of the trans-10,cis-12 and the cis-9,trans-11 isomer or an equal amount of a control fat supplement (Silafat, BASF) in which CLA were replaced by stearic acid (CON group). The trials were done in 2 subsequent years during the same season (autumnal calving) feeding a ration with the same components at equal portions. In trial 1, comprising 21 pluriparous cows, CLA was fed from d 1 to 182 of lactation and biopsies from s.c. fat around the tail head were collected at d -21, 1, 21, 70, 105, 182, 196, 224, and 252 relative to parturition from CON cows $(\mathrm{n}=11)$ and at d $-21,21,105,196$, and 252 from CLA cows. Details and outcomes in terms of performance, metabolite concentrations, and milk FFA profile from this experiment are described by Pappritz et al. (2011). In trial 2, 5 out of 25 primiparous Holstein cows (23 mo of age at parturition, on average) were slaughtered at 1 DIM and the remaining heifers were allocated randomly to either CON or CLA fat supplement starting from 1 DIM until slaughter. Five animals per group were slaughtered at 42 and 105 DIM, respectively. Samples from 3 v.c. AT (omental, mesenterial, and retroperitoneal) and 3 s.c. AT (tail head, withers, and sternum) were collected immediately after slaughter; all experimental details and main outcomes in terms of performance, adipose depot and organ weights, body composition, body fat mobilization, protein accretion, and energy utilization were reported by von Soosten et al. $(2011,2012)$ and of fat cell size by Akter et al. (2011).

Quantification of AQP7 mRNA using real-time PCR, tissue handling, reference gene (REF) selection, and basic PCR conditions were described previously (Saremi et al., 2012a,b). The primers used were 5'ATTGTGACTGGCATCCTTG'3 for forward and 5'TGGTTCTGAAGACTTGTGAG'3 for reverse (NCBI gene bank accession number: NM_001076378; fragment size: $139 \mathrm{bp}$ ). The PCR conditions were $10 \mathrm{~s}$ at $95^{\circ} \mathrm{C}$ for initial denaturation, $30 \mathrm{~s}$ at $95^{\circ} \mathrm{C}$ for denaturation, 35 $\mathrm{s}$ at $61^{\circ} \mathrm{C}$ for annealing, and $45 \mathrm{~s}$ at $72^{\circ} \mathrm{C}$ for extension. A PCR amplicon standard curve was used to estimate PCR efficiency per run. Two microliters of cDNA (diluted 1 to 4 ) as a template and $5 \mu \mathrm{L}$ of SYBR Green JumpStart Taq Readymix (Sigma-Aldrich, Steinheim, Germany) in a total volume of $10 \mu \mathrm{L}$ were run in an 


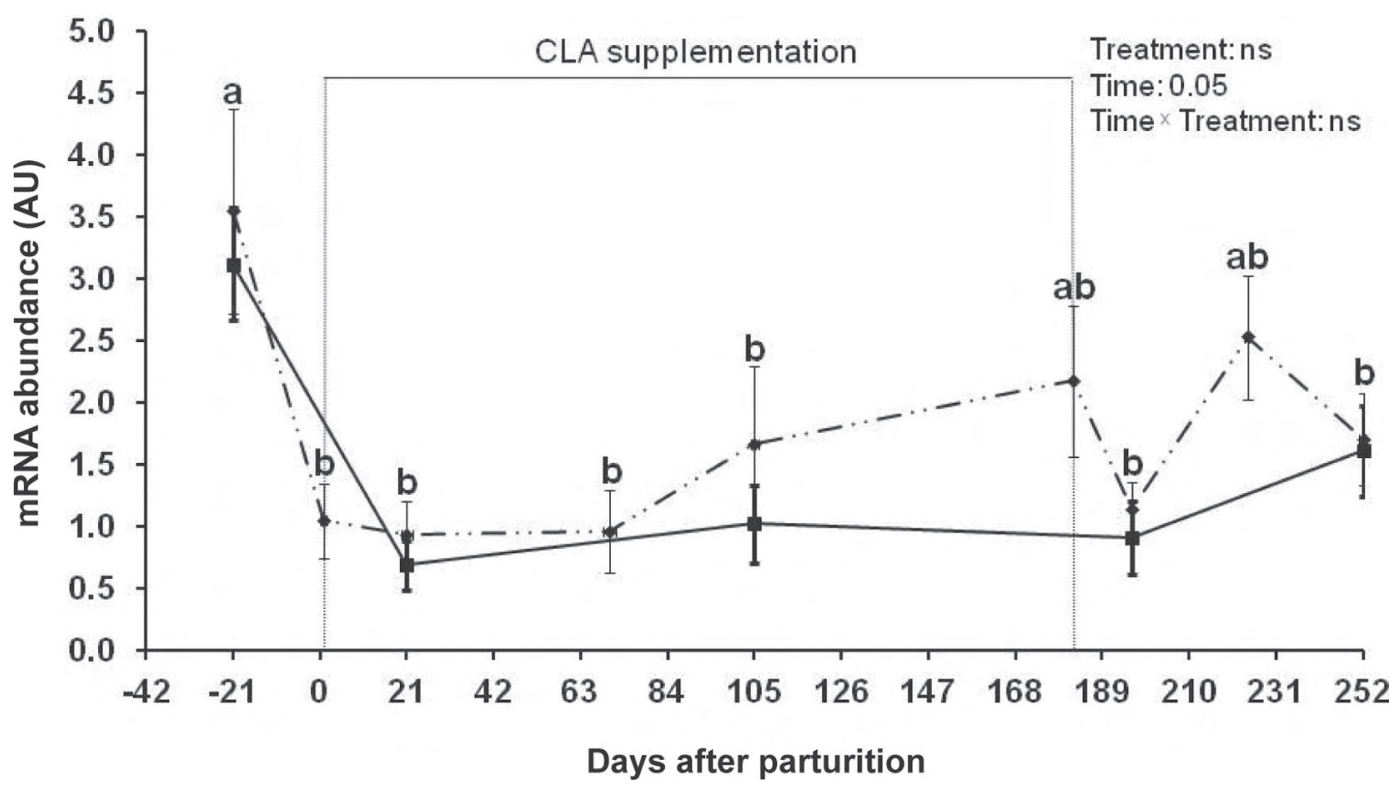

Figure 1. Aquaporin-7 (AQP7) mRNA abundance (mean $\pm \mathrm{SE}$ ) in subcutaneous tail head fat of multiparous cows receiving $100 \mathrm{~g} / \mathrm{d}$ of conjugated linoleic acids (CLA; Lutrell Pure, BASF SE, Ludwigshafen, Germany; n = 11; solid line) or a control fat supplement (Silafat, BASF $\mathrm{SE} ; \mathrm{n}=10$; dashed-dotted line) from d 1 to 182 postpartum. Samples from d 1, 70, 182, and 224 were restricted to the control cows. Treatment was not significant (ns); therefore the merged data were used to compare AQP7 mRNA abundance between different days. Different letters (a,b) indicate significant differences between days $(P<0.05)$. Data are normalized based on the geometric mean of 3 most stable reference genes (Saremi et al., 2012a). AU = arbitrary units.

Mx3000P (Agilent, Santa Clara, CA). For trial 1, the REF genes selected for s.c. AT were GAPDH, lipoprotein receptor related protein 10 (LRP10), and RNA polymerase II (POLR2A); for liver eucariotic translation initiation factor 3 (EIF3K), LRP10, and POLR2A were used. For trial 2, EIF3K, LRP10, POLR2A, Emerin, Marvel domain containing 1 and Hippocalcinlike 1 were used as REF genes. Normalization was done with the geometric mean of the respective set of REF (Saremi et al., 2012a,b).

All statistical analyses were performed using SPSS (version 20, SPSS Inc., Chicago, IL). For trial 1, the mixed model procedure was used. Treatments (CON and CLA) were considered as a fixed factor, sampling dates (time) as a repeated effect, and their respective interaction was included into the model. The covariance structure heterogeneous first-order autoregressive and Bonferroni correction were used. For trial 2, in which the repeated design was not applicable, all data were tested for homogeneity of variances $(P<0.1)$. Depending on homogeneity of variances, either the general linear model, with the fixed effects of treatments, dates, and the respective interaction, or nonparametric tests (Kruskal-Wallis or Mann-Whitney) were used. A $P$-value of $<0.05$ was considered significant.

In s.c. fat biopsies from pluriparous cows, AQP7 mRNA abundance decreased from 3 wk before calving to the day of parturition and remained at this level throughout the observation period until 252 DIM; no differences were detected between the cows from the CON and the CLA group (Figure 1). The peripartal decrease observed herein is in line with reports from murine 3T3-L1 adipocytes in which reduced AQP7 expression was observed upon long-term stimulation with $\beta$-adrenergic agonists used to stimulate lipolysis (Fasshauer et al., 2003; Rodríguez et al., 2011a). Moreover, with regard to decreased insulin serum concentrations and IS during early lactation, a decrease in AQP7 expression could be expected from earlier studies in human adipocytes reporting that insulin increases AQP7 expression (Rodríguez et al., 2011b). Contrasting results have been reported for murine adipocytes, in which insulin was shown to repress AQP7 gene expression (Kishida et al., 2001a; Kuriyama et al., 2002). In addition, the expression of AQP has been shown to be elevated in mouse and rat models of insulin resistance (Kuriyama et al., 2002; Nejsum et al., 2001). Species specificities in terms of the physiological relevance of glycerol for gluconeogenesis might account for these discrepancies; in negative energy balance, glycerol is the main substrate for gluconeogenesis in rodents, but this applies neither for humans, where it is pyruvate, nor for ruminants, where propionate ranks first as gluconeogenetic substrate throughout pregnancy and lactation (Aschenbach et al., 2010). A repression of AQP7 by the adipocyte-derived hormone leptin, as described for 
Table 1. Aquaporin-7 mRNA abundance (means \pm SEM) in different adipose tissue depots collected on 1, 42, and 105 DIM from primiparous cows receiving a control fat or a conjugated linoleic acid (CLA) supplement

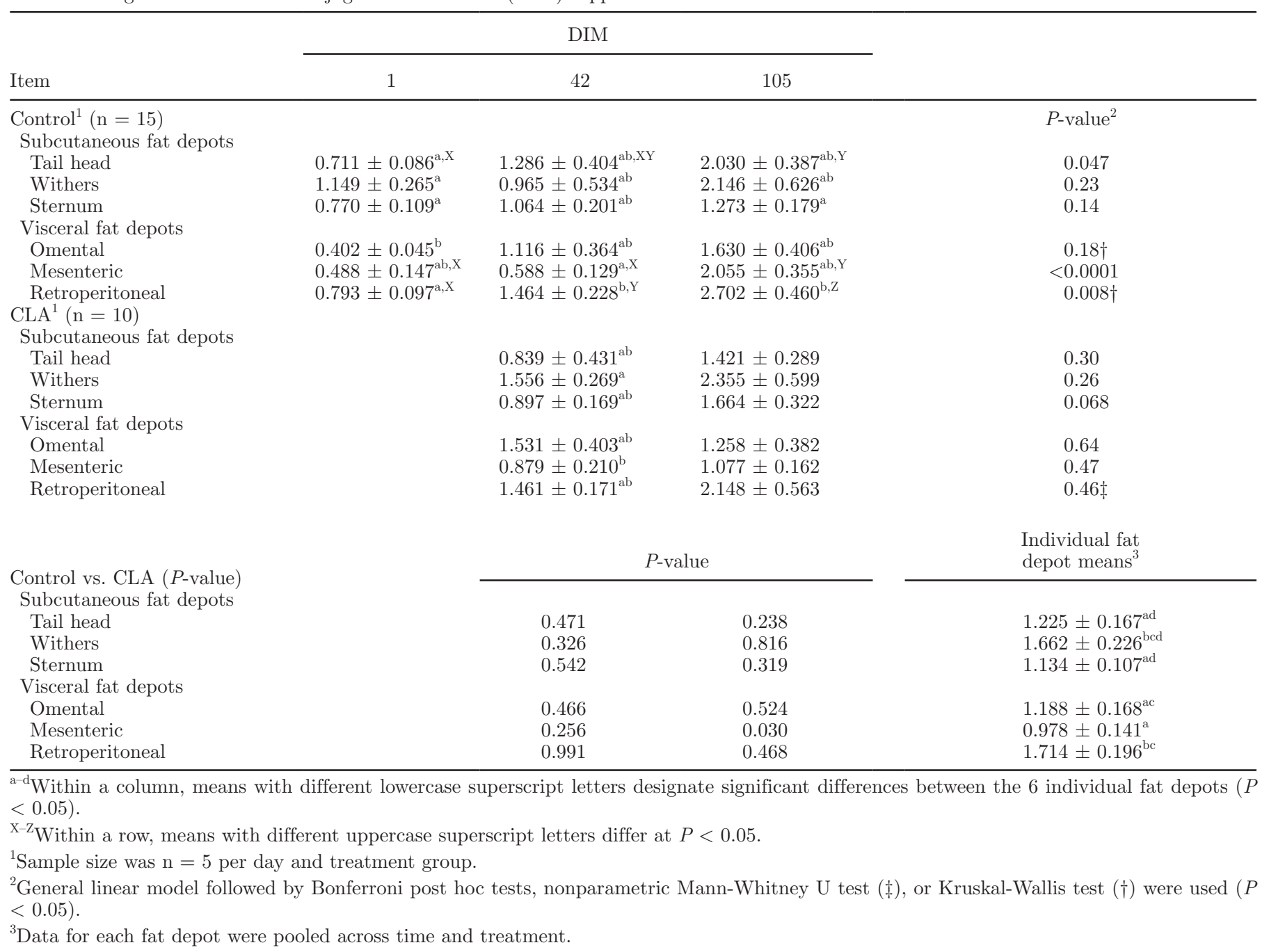

human adipocytes (Rodríguez et al., 2011b), could not be confirmed by our data, as the decrease of AQP7 expression toward parturition is knowingly accompanied by decreasing and not increasing leptin serum concentrations (e.g., Hachenberg et al., 2007); a concomitant decrease was also observed for the mRNA expression of its receptor (Saremi et al., 2012c). The increase of glycerol concentrations in blood around calving and the first wk thereafter parallels the rise of NEFA concentrations (Koltes and Spurlock, 2011). In the current study, plasma glycerol was not measured, but NEFA plasma concentrations returned to prepartum values within the first 2 wk of lactation (von Soosten et al., 2011). The AQP7 mRNA abundance actually remained low during almost the entire lactation period (i.e., far beyond the actual time of negative energy balance and decreased insulin resistance). Only at 182 and 224 DIM were the values reached not different from those of $3 \mathrm{wk}$ prepartum (samples at these DIM were obtained only from the CON group). Considering our data across the lactation cycle, and assuming that greater AQP7 mRNA abundance would translate to increased expression of the protein and would finally result in increased glycerol export from adipocytes, we speculate that the only situation during which the release of glycerol into the blood stream would be increased is late pregnancy and late lactation in pluriparous cows.

The AQP7 mRNA abundance in different fat depots obtained at slaughter from primiparous cows on 1, 42, and 105 DIM is shown in Table 1 . When comparing the different depots regardless of time and treatment, no general difference was observed between v.c. and s.c. fat depots; the lowest AQP7 mRNA values were observed in mesenterial and omental fat, but also in s.c. fat from the sternum and the tail head region. Retroperitoneal fat and s.c. fat from withers stood out with highest values. 
Differences in AQP7 gene expression are reported from human patients and point to overexpression in omental versus repressed expression in s.c. fat in obese subjects, as recently reviewed by Rodríguez et al. (2011a). In general, with increased duration of lactation, AQP7 mRNA abundance was numerically increased in most depots from the cows investigated herein (Table 1). Comparing 1 versus 105 DIM within the CON group, significant $(P<0.05)$ differences were established in the v.c. depots from mesenterial and retroperitoneal fat and in s.c. fat from the tail head; for omental and the 2 other s.c. depots (sternum and withers) the corresponding $P$-values, regardless of treatment, were $0.06,0.056$, and 0.121 , respectively. Comparing 1 against 42 DIM increased AQP7 mRNA values exclusively in retroperitoneal fat; this depot was also the only one for which a significant reduction of both depot mass and adipocyte size with DIM was established earlier (Akter et al., 2011; von Soosten et al., 2011). In AQP7-deficient mice, body fat mass is increased and adipocytes are greatly enlarged (Hara-Chikuma et al., 2005). Our data support a negative relationship between adipocyte size and AQP7 mRNA abundance for cows, at least in the retroperitoneal depot; both variables were inversely correlated $(\mathrm{r}=-0.414 ; P<0.05)$.

However, the increase of AQP7 mRNA with DIM observed in the primiparous cows is opposite that of findings in s.c. fat from pluriparous cows exhibiting constantly low AQP7 expression from 1 to 252 DIM. Accordingly, primiparous and pluriparous cows differ in several physiological and metabolic aspects: primiparous animals are still growing, whereas pluriparous cows are mature; the extent and duration of negative energy balance in pluriparous cows usually exceeds that of primiparous cows, and the concentration of metabolic hormones may also differ (e.g., primiparous cows have higher insulin concentrations during the first $7 \mathrm{wk}$ of lactation than pluriparous cows; Wathes et al., 2007). However, with regard to when AQP7 mRNA increased in the primiparous animals (105 DIM), gross metabolic differences can be ruled out, as all animals were in positive energy balance by that time.

Concerning potential CLA effects, AQP7 mRNA abundance was lower in mesenterial fat from CLAtreated cows as compared with CON cows at 105 DIM. In all other fat depots and in samples from 42 DIM, CLA and CON were not different. Based on the literature (Martins et al., 2010), we hypothesized that CLA might decrease AQP7 expression, however, the finding from mesenterial fat was isolated and a general mode of CLA action applying for all fat depots could not be confirmed.

Taken together, the longitudinal characterization of AQP7 mRNA expression in AT during lactation described herein yielded differences between primiparous and pluriparous cows, whereby primiparous cows showed increasing values within the first 105 DIM in contrast to pluriparous cows with constantly low values even beyond this time. Individual fat depots differed in terms of AQP7 mRNA abundance, but no general separation of s.c. and v.c. depots was observed. Changes in DIM tested in primiparous cows were consistent for all depots, whereas a decrease induced by CLA treatment was limited to the mesenterial depot.

\section{ACKNOWLEDGMENTS}

The animal experiments from which samples were used herein were funded by the German Research Foundation (DFG), Bonn, Germany (PAK 286/1: SA 432/10-1). We thank Inga Hofs for excellent technical assistance, and Sarah Winand, Iris Gockel-Böhner (all of Institute of Animal Science, University of Bonn, Germany) for their help during tissue sampling.

\section{REFERENCES}

Akter, S. H., S. Häussler, S. Dänicke, U. Müller, D. von Soosten, J. Rehage, and H. Sauerwein. 2011. Physiological and conjugated linoleic acid-induced changes of adipocyte size in different fat depots of dairy cows during early lactation. J. Dairy Sci. 94:2871-2882.

Aschenbach, J. R., N. B. Kristensen, S. S. Donkin, H. M. Hammon, and G. B. Penner. 2010. Gluconeogenesis in dairy cows: The secret of making sweet milk from sour dough. IUBMB Life 62:869-877.

Baumgard, L. H., B. A. Corl, D. A. Dwyer, and D. E. Bauman. 2002. Effects of conjugated linoleic acids (CLA) on tissue response to homeostatic signals and plasma variables associated with lipid metabolism in lactating dairy cows. J. Anim. Sci. 80:1285-1293.

Bell, A. W. 1995. Regulation of organic nutrient metabolism during transition from late pregnancy to early lactation. J. Anim. Sci. 73:2804-2819.

Bell, A. W., and D. E. Bauman. 1997. Adaptations of glucose metabolism during pregnancy and lactation. J. Mammary Gland Biol. Neoplasia 2:265-278.

Bennink, M. R., R. W. Mellenberger, R. A. Frobisch, and D. E. Bauman. 1972. Glucose oxidation and entry rate as affected by initiation of lactation. J. Dairy Sci. 55(Suppl. 1):172-173.

Bensinger, S. J., and P. Tontonoz. 2008. Integration of metabolism and inflammation by lipid-activated nuclear receptors. Nature 454:470-477.

Chilliard, Y. 1993. Dietary fat and adipose tissue metabolism in ruminants, pigs, and rodents: A review. J. Dairy Sci. 76:3897-3931.

Fasshauer, M., J. Klein, U. Lossner, M. Klier, S. Kralisch, and R. Paschke. 2003. Suppression of aquaporin adipose gene expression by isoproterenol, TNF alpha and dexamethasone. Horm. Metab. Res. 35:222-227.

Hachenberg, S., C. Weinkauf, S. Hiss, and H. Sauerwein. 2007. Evaluation of classification modes potentially suitable to identify metabolic stress in healthy dairy cows during the peripartal period. J. Anim. Sci. 85:1923-1932.

Hara-Chikuma, M., E. Sohara, T. Rai, M. Ikawa, M. Okabe, S. Sasaki, S. Uchida, and A. S. Verkman. 2005. Progressive adipocyte hypertrophy in aquaporin-7-deficient mice: Adipocyte glycerol permeability as a novel regulator of fat accumulation. J. Biol. Chem. 280:15493-15496.

Kay, J. K., J. R. Roche, C. E. Moore, and L. H. Baumgard. 2006. Effects of dietary conjugated linoleic acid on production and metabolic parameters in transition dairy cows grazing fresh pasture. J. Dairy Res. 73:367-377. 
Kishida, K., H. Kuriyama, T. Funahashi, I. Shimomura, S. Kihara, N. Ouchi, M. Nishida, H. Nishizawa, M. Matsuda, M. Takahashi, K. Hotta, T. Nakamura, S. Yamashita, Y. Tochino, and Y. Matsuzawa. 2000. Aquaporin adipose, a putative glycerol channel in adipocytes. J. Biol. Chem. 275:20896-20902.

Kishida, K., I. Shimomura, H. Kondo, H. Kuriyama, Y. Makino, H. Nishizawa, N. Maeda, M. Matsuda, N. Ouchi, S. Kihara, Y. Kurachi, T. Funahashi, and Y. Matsuzawa. 2001a. Genomic structure and insulin-mediated repression of the aquaporin adipose (AQPap), adipose-specific glycerol channel. J. Biol. Chem. 276:36251-36260.

Kishida, K., I. Shimomura, H. Nishizawa, N. Maeda, H. Kuriyama, H. Kondo, M. Matsuda, H. Nagaretani, N. Ouchi, K. Hotta, S. Kihara, T. Kadowaki, T. Funahashi, and Y. Matsuzawa. 2001b. Enhancement of the aquaporin adipose gene expression by a peroxisome proliferator-activated receptor gamma. J. Biol. Chem. 276:48572-48579.

Koltes, D. A., and D. M. Spurlock. 2011. Coordination of lipid dropletassociated proteins during the transition period of Holstein dairy cows. J. Dairy Sci. 94:1839-1848.

Kuriyama, H., I. Shimomura, K. Kishida, H. Kondo, N. Furuyama, H. Nishizawa, N. Maeda, M. Matsuda, H. Nagaretani, S. Kihara, T. Nakamura, Y. Tochino, T. Funahashi, and Y. Matsuzawa. 2002. Coordinated regulation of fat-specific and liver-specific glycerol channels, aquaporin adipose and aquaporin 9. Diabetes 51:29152921.

Lebeck, J., T. Østergård, A. Rojek, E.-M. Füchtbauer, S. Lund, S. Nielsen, and J. Praetorius. 2012. Gender-specific effect of physical training on AQP7 protein expression in human adipose tissue. Acta Diabetol. 49(Suppl. 1):S215-S226.

Maeda, N. 2012. Implications of aquaglyceroporins 7 and 9 in glycerol metabolism and metabolic syndrome. Mol. Aspects Med. 33:665-675.

Martins, A. P., P. A. Lopes, S. V. Martins, A. Madeira, N. C. Santos, J. A. M. Prates, T. F. Moura, and G. Soveral. 2010. Conjugated linoleic acid reduces permeability and fluidity of adipose plasma membranes from obese Zucker rats. Biochem. Biophys. Res. Commun. 398:199-204.

Nejsum, L. N., T. H. Kwon, D. Marples, A. Flyvbjerg, M. A. Knepper, J. Frokiaer, and S. Nielsen. 2001. Compensatory increase in AQP2, p-AQP2, and AQP3 expression in rats with diabetes mellitus. Am. J. Physiol. Renal Physiol. 280:F715-F726.

Pappritz, J., U. Meyer, R. Kramer, E. M. Weber, G. Jahreis, J. Rehage, G. Flachowsky, and S. Dänicke. 2011. Effects of long-term supplementation of dairy cow diets with rumen-protected conjugated linoleic acids (CLA) on performance, metabolic parameters and fatty acid profile in milk fat. Arch. Anim. Nutr. 65:89-107.
Reshef, L., Y. Olswang, H. Cassuto, B. Blum, C. M. Croniger, S. C. Kalhan, S. M. Tilghman, and R. W. Hanson. 2003. Glyceroneogenesis and the triglyceride/fatty acid cycle. J. Biol. Chem. 278:30413-30416.

Rodríguez, A., V. Catalán, J. Gómez-Ambrosi, and G. Frühbeck. 2011a. Aquaglyceroporins serve as metabolic gateways in adiposity and insulin resistance control. Cell Cycle 10:1548-1556.

Rodríguez, A., V. Catalán, J. Gómez-Ambrosi, S. Garcia-Navarro, F. Rotellar, V. Valenti, C. Silva, M. J. Gil, J. Salvador, M. A. Burrell, G. Calamita, M. M. Malagon, and G. Frühbeck. 2011b. Insulinand leptin-mediated control of aquaglyceroporins in human adipocytes and hepatocytes is mediated via the PI3K/Akt/mTOR signaling cascade. J. Clin. Endocrinol. Metab. 96:E586-E597.

Saremi, B., A. Al-Dawood, S. Winand, U. Müller, J. Pappritz, D. von Soosten, J. Rehage, S. Dänicke, S. Häußler, M. Mielenz, and H. Sauerwein. 2012a. Bovine haptoglobin as an adipokine: Serum concentrations and tissue expression in dairy cows receiving a conjugated linoleic acids supplement throughout lactation. Vet. Immunol. Immunopathol. 146:201-211.

Saremi, B., H. Sauerwein, S. Dänicke, and M. Mielenz. 2012b. Technical note: Identification of reference genes for gene expression studies in different bovine tissues focusing on different fat depots. J. Dairy Sci. 95:3131-3138.

Saremi, B., S. Winand, P. Friedrichs, H. Sauerwein, S. Dänicke, and M. Mielenz. 2012c. Adiponectin and leptin system: Long term physiological and conjugated linoleic acid induced changes. Book of Abstracts of the 63rd Annual Meeting of the European Association for Animal Production, 27.08.-31.08.2012, Bratislava, Slovakia, Page 64. Wageningen Academic Publishers, Wageningen, the Netherlands.

von Soosten, D., U. Meyer, M. Piechotta, G. Flachowsky, and S. Dänicke. 2012. Effect of conjugated linoleic acid supplementation on body composition, body fat mobilization, protein accretion, and energy utilization in early lactation dairy cows. J. Dairy Sci. 95:1222-1239.

von Soosten, D., U. Meyer, E. M. Weber, J. Rehage, G. Flachowsky, and S. Dänicke. 2011. Effect of trans-10, cis-12 conjugated linoleic acid on performance, adipose depot weights, and liver weight in early-lactation dairy cows. J. Dairy Sci. 94:2859-2870.

Wathes, D. C., Z. Cheng, N. Bourne, V. J. Taylor, M. P. Coffey, and S. Brotherstone. 2007. Differences between primiparous and multiparous dairy cows in the inter-relationships between metabolic traits, milk yield and body condition score in the periparturient period. Domest. Anim. Endocrinol. 33:203-225. 